\title{
A RESOLUÇÃO DO PROBLEMA DE DESPACHO ÓTIMO DE REATIVOS PELO MÉTODO DA FUNÇÃO LAGRANGIANA-BARREIRA RELAXADA
}

\author{
Vanusa Alves de Sousa* \\ Departamento de Engenharia Elétrica / EESC \\ Universidade de São Paulo (USP) \\ São Carlos - SP \\ vsousa@,sel.eesc.usp.br \\ Edméa Cássia Baptista \\ Departamento de Matemática / FC \\ Universidade Estadual Paulista (UNESP) \\ Bauru - SP

\section{Geraldo R. M. da Costa} \\ Departamento de Engenharia Elétrica / EESC \\ Universidade de São Paulo (USP) \\ São Carlos - SP \\ * Corresponding author / autor para quem as correspondências devem ser encaminhadas \\ Recebido em 05/2007; aceito em 02/2008 \\ Received May 2007; accepted February 2008
}

\section{Resumo}

Este artigo apresenta a aplicação do método da função Lagrangiana-barreira relaxada ao problema de despacho ótimo de reativos, o qual é um problema não-linear não convexo e de grande porte. Nesta abordagem as restrições de desigualdade são tratadas pela associação dos métodos de barreira modificada e primal-dual barreira logarítmica. Essas restrições são transformadas em igualdades através de variáveis auxiliares positivas e são relaxadas pelo parâmetro de barreira. Uma função Lagrangiana é associada ao problema modificado. As condições necessárias de primeira-ordem são aplicadas gerando um sistema não-linear o qual é resolvido pelo método de Newton. A relaxação das variáveis auxiliares resulta na expansão da região factível do problema original, permitindo atingir o limite das restrições de desigualdade. Testes numéricos nos sistemas CESP 53 barras, o equivalente brasileiro sul-sudeste e o teste comparativo com o método primal-dual barreira logarítmica indicam que o método apresentado é eficiente na resolução do problema de despacho ótimo de reativos.

Palavras-chave: programação não-linear; sistemas elétricos de potência; método primal-dual; método de Newton; relaxação das restrições.

\begin{abstract}
This work presents the application of the relaxed barrier-Lagrangian function method to the optimal reactive dispatch problem, which is a nonlinear nonconvex and large problem. In this approach the inequality constraints are treated by the association of modified barrier and primal-dual logarithmic barrier method. Those constraints are transformed in equalities through positive auxiliary variables and are perturbed by the barrier parameter. A Lagrangian function is associated to the modified problem. The first-order necessary conditions are applied generating a non-linear system which is solved by Newton's method. The auxiliary variables perturbation result in an expansion of the feasible set of the original problem, allowing the limits of the inequality constraints to be reach. Numeric tests with the systems CESP 53 buses and the south-southeast Brazilian and the comparative test with the primal-dual logarithmic barrier method indicate that presented method is efficient in the resolution of optimal reactive dispatch problem.
\end{abstract}

Keywords: nonlinear programming; power systems; primal-dual method; Newton's method; perturbation of constraints. 


\section{Introdução}

Desde a década de 80 , a reestruturação do setor elétrico vem ganhando muita atenção em todo o mundo. Antes deste período a estrutura organizacional do setor era vertical e monopolizada, ou seja, a geração, a transmissão e a distribuição de energia, eram controladas por uma "autoridade" conforme Christie et al. (2000). Com a desverticalização do setor e o surgimento do ambiente competitivo, as ferramentas de otimização passaram a desempenhar um importante papel para as empresas de energia.

No novo modelo do setor elétrico alguns problemas operacionais ainda não foram totalmente resolvidos. Pode-se citar, por exemplo, o problema de despacho ótimo de reativos (DOR) o qual é um problema de otimização não-linear e não convexo e de grande porte que determina um conjunto de variáveis ótimas de estado e controle da rede elétrica, a partir dos dados de carga e dos parâmetros do sistema elétrico de potência (SEP). O problema de DOR otimiza uma função objetivo enquanto satisfaz um conjunto de restrições físicas e operacionais impostas pelas limitações dos equipamentos e exigências de segurança. Pelo fato do DOR ser muito útil para análises e estudos nas atividades de operação e planejamento do SEP ele tem sido abordado por diversos pesquisadores, dos quais pode-se citar Huneault \& Galiana (1991), Momoh et al. (1999) e mais recentemente Baptista et al. (2006), entre outros.

$\mathrm{O}$ método de pontos interiores e suas variantes tem sido amplamente aplicados para resolver o problema de DOR como em Granville (1994), Costa (2002), Ramos et al. (2005), entre outros, devido a sua eficiência e robustez. Destaca-se que, na linha de pontos interiores para resolver problemas de programação não-linear ( $\mathrm{PNL}$ ) diversas abordagens têm sido propostas na literatura especializada, pode-se citar, entre outros, Breitfeld \& Shanno (1996), Shanno \& Vanderbei (2000), Forsgren et al. (2002), Byrd et al. (2003) e Akrotirianakis \& Rustem (2005).

Uma outra linha de técnicas para resolver problemas de PNL são os métodos Lagrangianos aumentados ou métodos de Multiplicadores e os métodos shifted-barrier como, por exemplo, Hestenes (1969), Powell (1969), Gill et al. (1988), Polyak (1992) e Conn et al. (1997). Ressalta-se o trabalho de Polyak (1992) o qual desenvolveu uma teoria de métodos da função barreira modificada (FBM) para resolver problemas de otimização restrita. Estes métodos combinam as melhores propriedades da função Lagrangiana clássica e da função barreira clássica (FBC). Em contraste com a FBC as FBM são definidas na solução; são suaves na vizinhança do ótimo; não vão para o infinito quando se aproximam do ótimo e representam explicitamente os multiplicadores de Lagrange. Além disso, a solução ótima encontrada no método da FBM pode, de fato, estar na fronteira da região factível, o que não acontece com a FBC, onde a solução somente pode estar próxima a fronteira, mas nunca alcançá-la. Uma característica interessante dos métodos baseados na FBM é de não precisarem de uma solução inicial factível, ao contrário dos métodos baseados na FBC que possuem essa desvantagem.

O objetivo deste trabalho é aplicar uma abordagem baseada na FBM e no método de pontos interiores para resolver o problema de DOR com função objetivo não-linear e restrições lineares e não-lineares. $\mathrm{O}$ método trata as restrições de desigualdade pelo método de barreira modificada, resultando em um problema equivalente modificado. Uma função Lagrangiana é associada a esse problema. As condições necessárias de primeira-ordem são aplicadas e obtém-se um sistema não-linear que é resolvido pelo método de Newton. A solução obtida pelo método de Newton fornece as direções de busca para atualização das variáveis primais e duais. Os multiplicadores de Lagrange associados à função barreira modificada são atualizados pela regra proposta por Polyak (1992). A abordagem será aplicada a um exemplo 
clássico e ao problema de DOR referente aos sistemas CESP 53 barras, o qual possui um total de 701 variáveis e 176 restrições sendo 96 de igualdade e 80 de desigualdade, e o equivalente brasileiro sul-sudeste o qual possui um total de 9025 variáveis e 2445 restrições sendo 1461 de igualdade e 984 de desigualdade.

Este trabalho está organizado da seguinte forma: na seção 2 apresenta-se a função barreira modificada, na seção 3 o método da função Lagrangiana-barreira relaxada aplicado ao problema de DOR, na seção 4 o algoritmo, na seção 5 os testes e resultados e finalmente na seção 6 as conclusões do trabalho.

\section{A Função Barreira Modificada (Polyak, 1992)}

A função barreira modificada é uma combinação da função Lagrangiana clássica e da função barreira clássica. Comparadas com as FBC, as FBM e suas derivadas são definidas na solução, não tendem para o infinito, são suaves na vizinhança do ponto ótimo e representam explicitamente as estimativas dos multiplicadores de Lagrange. Em contraste com a função Lagrangiana clássica, a FBM é estritamente convexa na vizinhança da solução. O método de barreira modificada transforma o problema restrito em um equivalente irrestrito, e resolve uma seqüência de problemas irrestritos até atingir o ótimo.

Em seu trabalho, Polyak, apresenta três tipos de FBM: uma para a FBC de Carrol, outra para a FBC de Frisch e a Função Barreira Shifted. Neste trabalho utiliza-se a função barreira modificada de Frisch, isto é, a logarítmica. Estas funções são definidas através da relaxação do conjunto de restrições factíveis.

Considere um problema de programação não-linear somente com restrições de desigualdade:

$$
\begin{aligned}
& \text { Minimizar } f(x) \\
& \text { sujeito a: } h_{j}(x) \geq 0, \quad j=1, \ldots, r
\end{aligned}
$$

em que $x \in \mathfrak{R}^{\mathrm{n}}$ representa o vetor das variáveis do problema e as funções $f$ e $h_{j}, j=1, \ldots, r$, são duas vezes continuamente diferenciáveis.

Aplica-se uma relaxação na restrição de desigualdade usando o parâmetro de barreira, $\mu$, e obtém-se um problema modificado, o qual é desenvolvido no Apêndice A.

\section{Minimizar $\mathrm{f}(\mathrm{x})$}

sujeito a: $\mu \ln \left(\mu^{-1} h_{j}(x)+1\right) \geq 0, \quad j=1, \ldots, r$

Ao problema (2) associa-se uma função Lagrangiana:

$$
L=f(x)-\mu \sum_{j=1}^{r} \rho_{j} \ln \left(\mu^{-1} h_{j}(x)+1\right),
$$

em que x pertence ao interior da região factível relaxada, isto é:

$$
\left\{\mathrm{x} \in \mathfrak{R}^{\mathrm{n}} \mid \mathrm{h}_{\mathrm{j}}(\mathrm{x}) \geq-\mu\right\}
$$

e $\rho_{\mathrm{j}}, \mathrm{j}=1, \ldots, \mathrm{r}$, são as estimativas não negativas dos multiplicadores de Lagrange na solução ótima. A função Lagrangiana (3) é denominada função barreira modificada. 
Aplicando-se as condições necessárias de primeira ordem na função (3), em relação à x, com $\rho_{\mathrm{j}}, \mathrm{j}=1, \ldots, \mathrm{r}$, e $\mu$ constantes, obtém-se:

$$
\nabla f(x)-\sum_{j=1}^{r}\left[\rho_{j} /\left(\mu^{-1} h(x)+1\right)\right] \nabla h(x)=0 .
$$

Aplica-se o método de Newton para resolver o sistema de equações não-lineares (5) e obtémse o seguinte sistema de forma simplificada:

$$
\nabla_{x x}^{2} L(x, \rho, \mu) \Delta x=-\nabla_{x} L(x, \rho, \mu)
$$

em que $\Delta \mathrm{x}$ é o vetor de busca.

$\mathrm{O}$ vetor $\mathrm{x}$ é atualizado por:

$$
x^{k+1}=x^{k}+\sigma \Delta x
$$

em que o tamanho do passo $\sigma>0$ é encontrado através da regra de Goldstein-Armijo conforme Nocedal \& Wright (1999).

As estimativas dos multiplicadores de Lagrange são atualizadas por meio da regra de Polyak (1992), a qual é desenvolvida no Apêndice B.

$$
\rho_{\mathrm{j}}^{\mathrm{k}+1}=\mu^{\mathrm{k}} \rho_{\mathrm{j}}^{\mathrm{k}} /\left(\mathrm{h}_{\mathrm{j}}\left(\mathrm{x}^{\mathrm{k}+1}\right)+\mu^{\mathrm{k}}\right)
$$

\section{O Método da Função Lagrangiana-Barreira Relaxada Aplicado ao Problema de DOR}

Considere o seguinte problema de DOR:

$$
\begin{array}{lll}
\text { Minimizar } & \mathrm{f}(\mathrm{x}) & \\
\text { sujeito a : } & \mathrm{g}_{\mathrm{i}}(\mathrm{x})=0 & \mathrm{i}=1,2, \ldots, \mathrm{m}<\mathrm{n} . \\
& \mathrm{h}_{\mathrm{j}}(\mathrm{x}) \geq 0 & \mathrm{j}=1,2, \ldots, \mathrm{r}
\end{array}
$$

$\mathrm{O}$ vetor $\mathrm{x} \in \mathfrak{R}^{\mathrm{n}}, \mathrm{x}=(\mathrm{t}, \mathrm{V}, \theta)$, representa as variáveis de estado e de controle do sistema, $\mathrm{t}$ os taps dos transformadores, $\mathrm{V}$ a magnitude da tensão e $\theta$ é o ângulo de fase da tensão. A função objetivo $\mathrm{f}(\mathrm{x})$ representa as perdas de potência ativa na transmissão; o vetor $\mathrm{g}(\mathrm{x})$ é o conjunto das equações de balanço do fluxo de potência e $\mathrm{h}(\mathrm{x})$ é o conjunto das restrições funcionais, que representam as gerações de potência reativa nas barras de controle de reativo e os limites nas magnitudes das tensões e dos transformadores. As funções $\mathrm{f}(\mathrm{x}), \mathrm{g}(\mathrm{x}) \mathrm{e} \mathrm{h}(\mathrm{x})$ do problema de DOR são apresentadas no Apêndice C.

Transforma-se a restrição de desigualdade do problema (7) em igualdade acrescentando-se uma variável auxiliar, como segue:

$$
\begin{array}{ccc}
\text { Minimizar } & f(x) & \\
\text { sujeito a : } & g_{i}(x)=0, & i=1, \ldots, m \\
& h_{j}(x)-s_{j}=0, & j=1, \ldots, r \\
s_{j} \geq 0, & j=1, \ldots, r
\end{array}
$$

sendo a variável $\mathrm{s}_{\mathrm{j}}, \mathrm{j}=1, \ldots, \mathrm{r}$, denominada de variável de excesso. 
Aplica-se uma relaxação na condição de não-negatividade do problema (8) usando o parâmetro de barreira, $\mu$. Essa relaxação resulta na expansão da região factível do problema original.

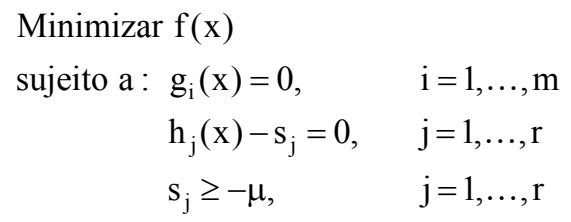

Como apresentado na seção 2, a função barreira modificada (Polyak, 1992) é utilizada para transformar o problema (9) no seguinte problema modificado:

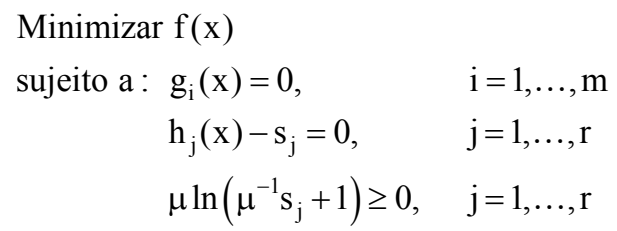

Associa-se ao problema (10) a seguinte função Lagrangiana:

$$
L(x, s, \lambda, \pi)=f(x)-\mu \sum_{j=1}^{r} \rho_{j} \ln \left(\mu^{-1} s_{j}+1\right)-\sum_{i=1}^{m} \lambda_{i} g_{i}(x)-\sum_{j=1}^{r} \pi_{j}\left(h_{j}(x)-s_{j}\right)
$$

em que $\rho_{\mathrm{j}}, \mathrm{j}=1, \ldots, \mathrm{r}$, são as estimativas dos multiplicadores de Lagrange, $\lambda$ e $\pi$ são os vetores dos multiplicadores de Lagrange associados as duas primeiras restrições.

As condições necessárias de primeira-ordem são aplicadas sobre função Lagrangiana (11), gerando um sistema de equações não-lineares, como segue:

$$
\left\{\begin{array}{l}
\nabla_{\mathrm{x}} \mathrm{L}(\mathrm{x}, \mathrm{s}, \lambda, \pi)=0 \\
\nabla_{\mathrm{s}} \mathrm{L}(\mathrm{x}, \mathrm{s}, \lambda, \pi)=0 \\
\nabla_{\lambda} \mathrm{L}(\mathrm{x}, \mathrm{s}, \lambda, \pi)=0 \\
\nabla_{\pi} \mathrm{L}(\mathrm{x}, \mathrm{s}, \lambda, \pi)=0
\end{array}\right.
$$

ou,

$$
\begin{cases}\nabla_{x} f(x)-J(x)^{T} \lambda-J_{1}(x)^{T} \pi=0 \\ -\rho /\left(\mu^{-1} s_{j}+1\right)+\pi_{j}=0, & j=1, \ldots, r \\ -g_{i}(x)=0, & i=1, \ldots, m \\ -\left(h_{j}(x)-s_{j}\right)=0, & j=1, \ldots, r\end{cases}
$$

em que: $J(x)^{T}=\left(\nabla_{x} g_{1}(x), \ldots, \nabla_{x} g_{m}(x)\right), e J_{1}(x)^{T}=\left(\nabla_{x} h_{1}(x), \nabla_{x} h_{2}(x), \ldots, \nabla_{x} h_{r}(x)\right)$.

O sistema de equações não-lineares (13) é resolvido utilizando-se o método de Newton. A aplicação do método de Newton gera as direções de busca $(\Delta x, \Delta s, \Delta \lambda, \Delta \pi)$, as quais serão utilizadas para a atualização das variáveis do problema, e resulta num sistema matricial, que, pode ser representado de forma simplificada como:

$$
\mathrm{W} \Delta \mathrm{d}=-\nabla \mathrm{L}
$$


em que:

$$
\mathrm{W}=\left(\begin{array}{cccc}
\nabla_{\mathrm{xx}} \mathrm{L} & -\mathrm{I} & -\mathrm{J}(\mathrm{x})^{\mathrm{T}} & -\mathrm{J}_{1}(\mathrm{x})^{\mathrm{T}} \\
-\mathrm{I} & \mu \mathrm{S} & 0 & -\mathrm{I} \\
-\mathrm{J}(\mathrm{x}) & 0 & 0 & 0 \\
-\mathrm{J}_{1}(\mathrm{x}) & -\mathrm{I} & 0 & 0
\end{array}\right),
$$

é a matriz Hessiana da função Lagrangiana com:

$$
S=\left(\begin{array}{ccc}
\rho /\left(\mu^{-1} \mathrm{~s}_{1}+1\right)^{2} & & 0 \\
& \ddots & \\
0 & & \rho /\left(\mu^{-1} \mathrm{~s}_{\mathrm{r}}+1\right)^{2}
\end{array}\right),
$$

$\Delta \mathrm{d}^{\mathrm{T}}=(\Delta \mathrm{x}, \Delta \mathrm{s}, \Delta \lambda, \Delta \pi) \mathrm{e}$

$$
\nabla L=\left[\begin{array}{ll}
\nabla_{x} f(x)-J(x)^{T} \lambda-J_{1}(x)^{T} \pi \\
-\rho /\left(\mu^{-1} s_{j}+1\right)+\pi_{j} & j=1, \ldots, r \\
-g_{i}(x) & i=1, \ldots, m \\
-\left(h(x)-s_{j}\right) & j=1, \ldots, r
\end{array}\right]
$$

é o vetor gradiente. Verifica-se que o sistema continua determinado para $\mathrm{s}_{\mathrm{j}}=0, \mathrm{j}=1, \ldots, \mathrm{r}$.

Os vetores das variáveis $\mathrm{x}, \mathrm{s}, \lambda$ e $\pi$ são atualizados da seguinte forma:

$$
\begin{aligned}
& x^{k+1}=x^{k}+\alpha_{p} \Delta x^{k} \\
& s^{k+1}=s^{k}+\alpha_{p} \Delta s^{k} \\
& \lambda^{k+1}=\lambda^{k}+\alpha_{d} \Delta \lambda^{k} \\
& \pi^{k+1}=\pi^{k}+\alpha_{d} \Delta \pi^{k}
\end{aligned}
$$

em que $\alpha_{p}$ e $\alpha_{d}$ são os passos utilizados na atualização das variáveis primais e duais, respectivamente.

Neste trabalho adotou-se para o cálculo do passo máximo a estratégia utilizada por Monteiro \& Adler (1989 a,b). O passo é obtido encontrando o menor elemento positivo entre o mínimo tamanho de passo de todos os componentes do vetor da variável auxiliar e este menor elemento é comparado ao valor 1. Escolhe-se o menor entre eles. O objetivo do passo primal é manter as variáveis primais dentro dos seus limites. O passo dual é calculado de forma que cada componente do vetor dual $\pi$ permaneça com seu respectivo sinal, isto é:

$$
\begin{aligned}
& \alpha_{\mathrm{p}}=\min \left(\min _{\Delta \mathrm{s}<0, \mathrm{~s}>0} \frac{\mathrm{s}}{|\Delta \mathrm{s}|}, 1\right), \\
& \alpha_{d}=\min \left(\min _{\Delta \pi<0} \frac{\pi}{|\Delta \pi|}, 1\right) .
\end{aligned}
$$


O parâmetro de barreira $\mu$ é atualizado segundo Melman \& Polyak (1996), por:

$$
\mu^{\mathrm{k}+1}=\mu^{\mathrm{k}}(1-\delta / \sqrt{\mathrm{r}}),
$$

em que r é o número de restrições de desigualdade do problema e

$$
\delta=\max \left(1 /\left(\mu^{-1} s_{j}+1\right)\right), \quad j=1, \ldots, r \text { para } s_{j}>0 .
$$

As estimativas dos multiplicadores de Lagrange $\rho$ são atualizadas pela regra de Polyak (1992), da seguinte forma:

$$
\rho_{\mathrm{j}}^{\mathrm{k}+1}=\mu^{\mathrm{k}+1} \rho_{\mathrm{j}}^{\mathrm{k}} /\left(\mathrm{s}_{\mathrm{j}}^{\mathrm{k}+1}+\mu^{\mathrm{k}+1}\right) .
$$

\section{Algoritmo}

O método da função Lagrangiana-barreira relaxada pode ser apresentado pelo seguinte algoritmo:

1. Dado o problema (7), construa a função Lagrangiana (11);

2. Faça $\mathrm{k}=0$ e dê uma estimativa inicial para $\mathrm{d}^{\mathrm{k}}=\left(\mathrm{x}^{\mathrm{k}}, \mathrm{s}^{\mathrm{k}}, \lambda^{\mathrm{k}}, \pi^{\mathrm{k}}\right), \rho^{\mathrm{k}}>0$, e $\mu^{\mathrm{k}}>0$;

3. Determine o gradiente e a matriz Hessiana da função Lagrangiana, e resolva o sistema (14);

4. Calcule os passos primais e duais. Atualize as variáveis do problema. Faça $\mathrm{k}=\mathrm{k}+1$;

5. Atualize o parâmetro de barreira $\mu$ e as estimativas dos multiplicadores de Lagrange, $\rho$, utilizando (18) e (19) respectivamente.

6. Se o critério de parada está satisfeito então pare. Caso contrário vá para o passo 3 ;

Um ponto inicial factível para o problema original não é obrigatório, mas a condição $s>-\mu$ deve ser satisfeita e na solução $s \geq 0$ e $\pi \geq 0$.

O critério de parada é um teste para verificar a convergência do algoritmo, da seguinte forma:

$$
\begin{array}{lll}
v_{1}^{\mathrm{k}} \leq \xi_{1} & & \left\|\mathrm{~g}\left(\mathrm{x}^{\mathrm{k}}\right)\right\|_{\infty} \leq \xi_{1} \\
v_{2}^{\mathrm{k}} \leq \xi_{2} & \text { ou } & v_{2}^{\mathrm{k}} \leq \xi_{2} \\
v_{3}^{\mathrm{k}} \leq \xi_{3} & &
\end{array}
$$

em que:

$$
\begin{aligned}
& v_{1}=\|\mathrm{g}(\mathrm{x})\|_{\infty}, \\
& v_{2}=\left|\mathrm{f}\left(\mathrm{x}^{\mathrm{k}}\right)-\mathrm{f}\left(\mathrm{x}^{\mathrm{k}-1}\right)\right| /\left(1+\left|\mathrm{f}\left(\mathrm{x}^{\mathrm{k}}\right)\right|\right), \\
& v_{3}=\max \left\{\mathrm{h}_{\mathrm{j}}(\mathrm{x})\right\}, \mathrm{j}=1, \ldots, \mathrm{r} .
\end{aligned}
$$

Se os critérios $v_{1}^{\mathrm{k}} \leq \xi_{1}, v_{2}^{\mathrm{k}} \leq \xi_{2}$ e $v_{3}^{\mathrm{k}} \leq \xi_{3}$ estão satisfeitos, então a factibilidade primal e dual escalar estão garantidas, o que significa que na iteração $\mathrm{k}$ tem-se uma solução que satisfaz as condições de Karush-Kuhn-Tucker (KKT) com precisão $\xi_{1}$. Quando problemas numéricos 
impedem a verificação destas, o algoritmo pára assim que a factibilidade das restrições de igualdade é alcançada e as mudanças no valor da função objetivo e das variáveis são desprezíveis. Valores típicos para as tolerâncias são $\xi_{1}=10^{-3}, \xi_{2}=10^{-2} \mathrm{e} \xi_{3}=10^{-1}$.

\section{Testes e Resultados}

Para comprovar a eficiência do método da função Lagrangiana-barreira relaxada (FLBR), este foi aplicada a um exemplo apresentado por Baptista et al. (2004), ao problema de DOR referente aos sistemas CESP 53 barras e equivalente brasileiro sul-sudeste e comparado ao método primal-dual barreira logarítmica (PDBL), este último e sua implementação computacional são apresentados em Sousa et al. (2004). No exemplo ilustrativo são apresentados dois testes com o método da FLBR, um com o ponto inicial interior e outro com ponto inicial exterior ao conjunto factível. No problema de DOR a função objetivo utilizada foi a de perdas de potência ativa na transmissão que é uma função não-linear, não convexa e não separável. A implementação computacional foi realizada em linguagem Fortran.

\section{A - Exemplo Ilustrativo}

Considere o seguinte problema de PNL:

$$
\begin{array}{cl}
\text { Minimizar } & \left(\mathrm{x}_{1}-2\right)^{4}+\left(\mathrm{x}_{1}-2 \mathrm{x}_{2}\right)^{2} \\
\text { sujeito a: } & \mathrm{x}_{1}+\mathrm{x}_{2}-3=0 \\
& \mathrm{x}_{1}^{2}-\mathrm{x}_{2} \leq 0 \\
& 1,5 \leq \mathrm{x}_{2} \leq 2
\end{array}
$$

A função Lagrangiana associada ao problema (20) é:

$$
\begin{aligned}
\mathrm{L}\left(\mathrm{x}_{1}, \mathrm{x}_{2}, \mathrm{~s}, \lambda, \pi\right) & =\left(\mathrm{x}_{1}-2\right)^{4}+\left(\mathrm{x}_{1}-2 \mathrm{x}_{2}\right)^{2}-\mu \rho_{1} \ln \left(\mu^{-1} \mathrm{~s}_{1}+1\right)-\mu \rho_{2} \ln \left(\mu^{-1} \mathrm{~s}_{2}+1\right) \\
& -\mu \rho_{3} \ln \left(\mu^{-1} \mathrm{~s}_{3}+1\right)-\lambda\left(\mathrm{x}_{1}+\mathrm{x}_{2}-3\right)-\pi_{1}\left(\mathrm{x}_{1}^{2}-\mathrm{x}_{2}+\mathrm{s}_{1}\right)- \\
& \pi_{2}\left(\mathrm{x}_{2}+\mathrm{s}_{2}-2\right)-\pi_{3}\left(\mathrm{x}_{2}-\mathrm{s}_{3}-1,5\right)
\end{aligned}
$$

As condições necessárias de primeira-ordem são aplicadas à função Lagrangiana (21), gerando um sistema de equações não-lineares, como segue:

$$
\nabla \mathrm{L}\left(\mathrm{x}_{1}, \mathrm{x}_{2}, \mathrm{~s}_{1}, \mathrm{~s}_{2}, \mathrm{~s}_{3}, \lambda, \pi_{1}, \pi_{2}, \pi_{3}\right)=0
$$

em que:

$$
\nabla \mathrm{L}=\left[\begin{array}{c}
4\left(\mathrm{x}_{1}-2\right)^{3}+2\left(\mathrm{x}_{1}-2 \mathrm{x}_{2}\right)-\lambda-2 \mathrm{x}_{1} \pi_{1} \\
-4\left(\mathrm{x}_{1}-2 \mathrm{x}_{2}\right)-\lambda-\pi_{1}-\pi_{2}-\pi_{3} \\
-\rho_{1} /\left(\mu^{-1} \mathrm{~s}_{1}+1\right)-\pi_{1} \\
-\rho_{2} /\left(\mu^{-1} \mathrm{~s}_{2}+1\right)-\pi_{2} \\
\rho_{3} /\left(\mu^{-1} \mathrm{~s}_{3}+1\right)-\pi_{3} \\
-\left(\mathrm{x}_{1}+\mathrm{x}_{2}-3\right) \\
-\left(\mathrm{x}_{1}^{2}-\mathrm{x}_{2}+\mathrm{s}_{1}\right) \\
-\left(\mathrm{x}_{2}+\mathrm{s}_{2}-2\right) \\
-\left(\mathrm{x}_{2}-\mathrm{s}_{3}-1,5\right)
\end{array}\right]
$$


O método de Newton é aplicado ao sistema de equações não-lineares (22) para determinar os fatores de correção das variáveis $\Delta \mathrm{x}_{1}, \Delta \mathrm{x}_{2}, \Delta \mathrm{s}_{1}, \Delta \mathrm{s}_{2}, \Delta \mathrm{s}_{3}, \Delta \lambda, \Delta \pi_{1}, \Delta \pi_{2}$ e $\Delta \pi_{3}$ resultando em:

$\mathrm{W} \Delta \mathrm{d}=-\nabla \mathrm{L}$

em que:

$$
\Delta \mathrm{d}=\left(\Delta \mathrm{x}_{1}, \Delta \mathrm{x}_{2}, \Delta \mathrm{s}_{1}, \Delta \mathrm{s}_{2}, \Delta \mathrm{s}_{3}, \Delta \lambda, \Delta \pi_{1}, \Delta \pi_{2}, \Delta \pi_{3}\right),
$$

W é a matriz Hessiana da função Lagrangiana, dada por:

$$
\mathrm{W}=\left[\begin{array}{ccccccccc}
\nabla_{\mathrm{x}_{1} \mathrm{x}_{1}}^{2} \mathrm{~L} & \nabla_{\mathrm{x}_{1} \mathrm{x}_{2}}^{2} \mathrm{~L} & 0 & 0 & 0 & -1 & -2 \mathrm{x}_{1} & 0 & 0 \\
\nabla_{\mathrm{x}_{2} \mathrm{x}_{1}}^{2} \mathrm{~L} & \nabla_{\mathrm{x}_{2} \mathrm{x}_{2}}^{2} \mathrm{~L} & 0 & 0 & 0 & -1 & 1 & -1 & -1 \\
0 & 0 & -\mu \rho_{1} /\left(\mu^{-1} \mathrm{~s}_{1}+1\right)^{2} & 0 & 0 & 0 & -1 & 0 & 0 \\
0 & 0 & 0 & -\mu \rho_{2} /\left(\mu^{-1} \mathrm{~s}_{2}+1\right)^{2} & 0 & 0 & 0 & -1 & 0 \\
0 & 0 & 0 & 0 & \mu \rho_{3} /\left(\mu^{-1} \mathrm{~s}_{3}+1\right)^{2} & 0 & 0 & 0 & 1 \\
-1 & -1 & 0 & 0 & 0 & 0 & 0 & 0 & 0 \\
-2 \mathrm{x}_{1} & 1 & -1 & 0 & 0 & 0 & 0 & 0 & 0 \\
0 & -1 & 0 & -1 & 0 & 0 & 0 & 0 & 0 \\
0 & -1 & 0 & 0 & 1 & 0 & 0 & 0 & 0
\end{array}\right]
$$

com:

$$
\begin{aligned}
& \nabla_{\mathrm{x}_{1} \mathrm{x}_{1}}^{2} \mathrm{~L}=12\left(\mathrm{x}_{1}-2\right)^{2}+2-2 \pi_{1} \\
& \nabla_{\mathrm{x}_{1} \mathrm{x}_{2}}^{2} \mathrm{~L}=\nabla_{\mathrm{x}_{2} \mathrm{x}_{1}}^{2}=-4 \\
& \nabla_{\mathrm{x}_{2} \mathrm{x}_{2}}^{2} \mathrm{~L}=8
\end{aligned}
$$

Os vetores das variáveis $\mathrm{x}, \mathrm{s}, \lambda \mathrm{e} \pi$ são atualizados usando (15). O parâmetro de barreira $\mu \mathrm{e}$ o vetor das estimativas dos multiplicadores de Lagrange, $\rho$, são atualizados utilizando (18) e (19), respectivamente.

A Tabela 1 apresenta o processo de convergência do método da função Lagrangiana-barreira relaxada aplicado ao problema (20), para um ponto inicial factível. Na Tabela 1, It. é o número da iteração, F. Ob. é o valor da função objetivo. Os valores iniciais: das variáveis de folga $\mathrm{s}_{1}=-\mathrm{x}_{1}{ }^{2}+\mathrm{x}_{2} ; \mathrm{s}_{2}=-\mathrm{x}_{2}+2 ; \mathrm{s}_{3}=\mathrm{x}_{2}-1,50$ e dos multiplicadores de Lagrange associados às restrições de igualdade e desigualdade, $\lambda=0 ; \quad \pi_{1}=-\rho_{1} /\left(\left(\mathrm{s}_{1} / \mu\right)+1\right) ; \quad \pi_{2}=-\rho_{2} /\left(\left(\mathrm{s}_{2} / \mu\right)+1\right)$; $\pi_{3}=\rho_{3} /\left(\left(s_{3} / \mu\right)+1\right)$, respectivamente. As equações para calcular os valores iniciais de $s_{1}, s_{2}, s_{3}$, $\pi_{1}, \pi_{2}$ e $\pi_{3}$ são obtidas do sistema (13). Neste exemplo as tolerâncias foram de $\xi_{1}=10^{-4}$, $\xi_{2}=10^{-2} \mathrm{e} \xi_{3}=10^{-1}$.

A Tabela 2 mostra os valores dos multiplicadores de Lagrange durante o processo de otimização. 
Tabela 1 - Convergência do método da FLBR aplicado ao problema (20).

\begin{tabular}{||c||c||c|c|c|c|c|c|c||c|}
\hline \hline It. & F. Ob. & $\mathbf{x}_{\mathbf{1}}$ & $\mathbf{x}_{\mathbf{2}}$ & $\mathbf{s}_{\mathbf{1}}$ & $\mathbf{s}_{\mathbf{2}}$ & $\mathbf{s}_{\mathbf{3}}$ & $\boldsymbol{\mu}$ & $\boldsymbol{\alpha}_{\mathbf{p}}$ & $\boldsymbol{\alpha}_{\mathbf{d}}$ \\
\hline \hline 0 & 7,950 & 1,100 & 1,900 & 0,690 & 0,100 & 0,400 & 0,950 & - & - \\
\hline \hline 1 & 4,435 & 1,316 & 1,684 & $-9,31 \mathrm{e}-10$ & 0,316 & 0,184 & 0,491 & 0,806 & 1,000 \\
\hline 2 & 4,610 & 1,303 & 1,697 & $-2,65 \mathrm{e}-4$ & 0,303 & 0,197 & 0,289 & 1,000 & 1,000 \\
\hline \hline 3 & 4,612 & 1,303 & 1,697 & $1,406 \mathrm{e}-4$ & 0,303 & 0,197 & 0,190 & 1,000 & 1,000 \\
\hline
\end{tabular}

Tabela 2 - Valores dos multiplicadores de Lagrange durante o processo de otimização.

\begin{tabular}{|c||c||c||c||c||c||c|c||}
\hline \hline It. & $\lambda$ & $\pi_{1}$ & $\pi_{\mathbf{2}}$ & $\pi_{\mathbf{3}}$ & $\rho_{\mathbf{1}}$ & $\rho_{\mathbf{2}}$ & $\rho_{\mathbf{3}}$ \\
\hline \hline 0 & 0 & $-2,230$ & $-0,005$ & 0,007 & 3,850 & 0,006 & 0,010 \\
\hline \hline 1 & 4,303 & $-3,281$ & $-0,004$ & 0,008 & 3,850 & 0,004 & 0,007 \\
\hline \hline 2 & 4,512 & $-3,851$ & $-0,002$ & 0,005 & 3,854 & 0,002 & 0,004 \\
\hline \hline 3 & 4,509 & $-3,857$ & $-0,001$ & 0,003 & 3,851 & 0,001 & 0,002 \\
\hline
\end{tabular}

Observa-se que o problema convergiu em 3 iterações com a restrição de desigualdade $\left(\mathrm{x}_{1}^{2}-\mathrm{x}_{2}-\mathrm{s}_{1} \leq 0\right)$ ativa na solução.

Para uma melhor visualização do processo de convergência do método para o problema (20), apresenta-se a Figura 1. Verifica-se na Figura 1, que a partir de um ponto inicial factível, o método procura um ponto na região factível relaxada, a seguir retorna a região factível e caminha pelo interior até atingir a solução ótima do problema, mantendo satisfeitas a restrição de igualdade e a restrição canalizada da variável $x_{2}$ durante todo o processo de otimização.

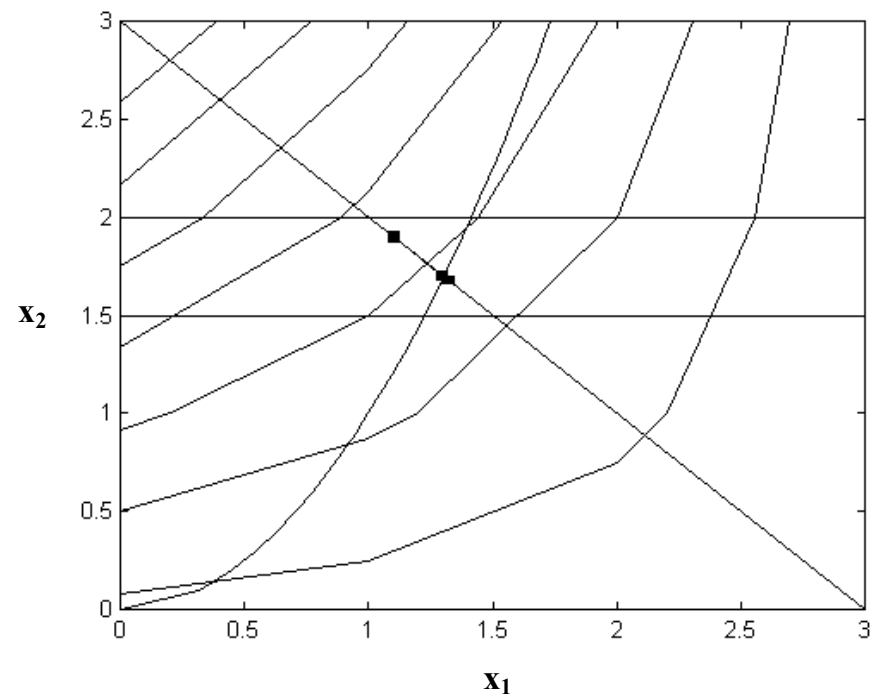

Figura 1 - Convergência para o ponto inicial na região factível.

A Tabela 3 apresenta o processo de convergência do método da função FLBR aplicado ao problema (20), para um ponto inicial infactível, isto é, na região factível relaxada. Neste exemplo as tolerâncias foram de $\xi_{1}=10^{-4}, \xi_{2}=10^{-2}$ e $\xi_{3}=10^{-1}$. 
Tabela 3 - Convergência do método FLBR aplicado ao problema (20).

\begin{tabular}{|c||c|c||c|c|c||c||c||c|c||}
\hline \hline It. & F. Ob. & $\mathbf{x}_{\mathbf{1}}$ & $\mathbf{x}_{\mathbf{2}}$ & $\mathbf{s}_{\mathbf{1}}$ & $\mathbf{s}_{\mathbf{2}}$ & $\mathbf{s}_{\mathbf{3}}$ & $\boldsymbol{\mu}$ & $\boldsymbol{\alpha}_{\mathbf{p}}$ & $\boldsymbol{\alpha}_{\mathbf{d}}$ \\
\hline \hline 0 & 11,586 & 1,600 & 2,500 & $-0,060$ & $-0,500$ & 1,000 & 1,600 & - & - \\
\hline \hline 1 & 4,422 & 1,324 & 1,676 & $-7,20 \mathrm{e}-4$ & 0,324 & 0,176 & 0,768 & 1,000 & 0,521 \\
\hline \hline 2 & 4,611 & 1,303 & 1,697 & $4,24 \mathrm{e}-4$ & 0,303 & 0,197 & 0,415 & 1,000 & 1,000 \\
\hline \hline 3 & 4,611 & 1,303 & 1,697 & $-7,50 \mathrm{e}-12$ & 0,303 & 0,197 & 0,190 & 0,151 & 1,000 \\
\hline
\end{tabular}

A Tabela 4 mostra os valores dos multiplicadores de Lagrange durante o processo de otimização.

Tabela 4 - Valores dos multiplicadores de Lagrange durante o processo de otimização.

\begin{tabular}{||c||c||c||c||c||c||c||c||}
\hline \hline It. & $\boldsymbol{\lambda}$ & $\boldsymbol{\pi}_{\mathbf{1}}$ & $\boldsymbol{\pi}_{\mathbf{2}}$ & $\boldsymbol{\pi}_{\mathbf{3}}$ & $\boldsymbol{\rho}_{\mathbf{1}}$ & $\boldsymbol{\rho}_{\mathbf{2}}$ & $\boldsymbol{\rho}_{\mathbf{3}}$ \\
\hline \hline 0 & 0 & $-4,000$ & $-0,007$ & 0,006 & 3,850 & 0,005 & 0,010 \\
\hline \hline 1 & 2,341 & $-3,794$ & $-0,000$ & 0,009 & 3,854 & 0,003 & 0,008 \\
\hline \hline 2 & 4,509 & $-3,854$ & $-0,003$ & 0,006 & 3,850 & 0,002 & 0,006 \\
\hline \hline 3 & 4,506 & $-3,850$ & $-0,001$ & 0,004 & 3,850 & 0,001 & 0,003 \\
\hline
\end{tabular}

Observa-se que o problema convergiu em 3 iterações com a restrição de desigualdade $\left(\mathrm{x}_{1}^{2}-\mathrm{x}_{2}-\mathrm{s}_{1} \leq 0\right)$ ativa na solução. A Figura 2 apresenta o processo de convergência do método para os dados da Tabela 3, nota-se um comportamento semelhante ao da Figura 1 com exceção do ponto inicial.

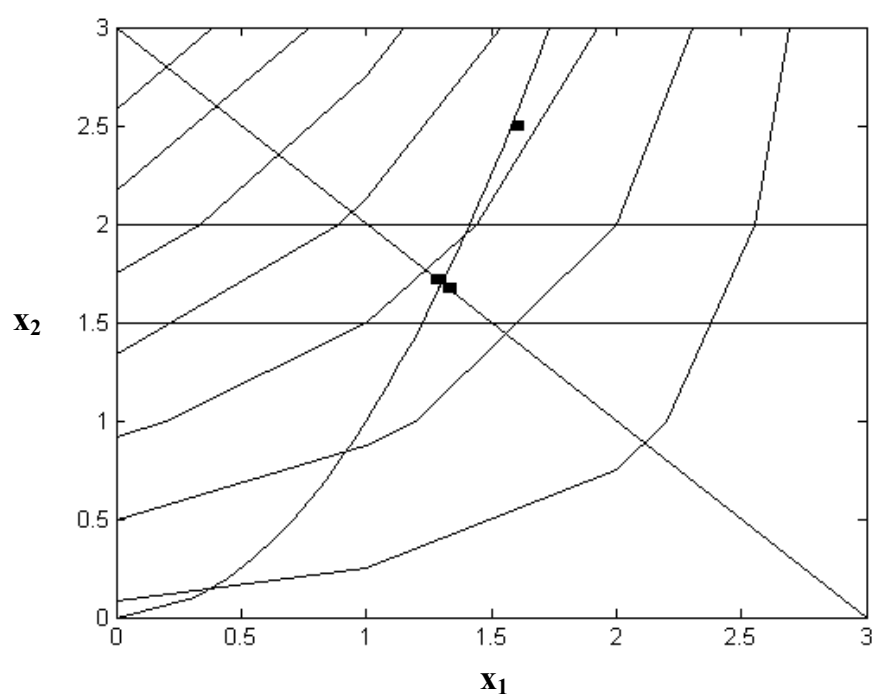

Figura 2 - Convergência para o ponto inicial na região factível relaxada.

\section{B - Sistema CESP 53 Barras}

O sistema brasileiro equivalente CESP $440 \mathrm{KV}$ de geração e transmissão com 53 barras possui as seguintes características: 1 barra de referência, 8 barras de controle de reativos, 44 barras de carga, 65 linhas de transmissão e 19 transformadores com taps variáveis. 
O problema de DOR associado ao sistema CESP 53 barras é composto por uma função objetivo, 96 equações não-lineares, 8 inequações não-lineares canalizadas de potência reativa, 19 restrições canalizadas de tap e 53 restrições canalizadas de tensão. Com um total de 701 variáveis e uma matriz Hessiana da função Lagrangiana de ordem 540.

A Tabela 5 apresenta um resumo do processo de convergência do método FLBR, com o número da iteração, o valor da função objetivo, a maior violação das restrições de igualdade ( Erro $\left.=\max \left\|\mathrm{g}_{\mathrm{i}}(\mathrm{x})\right\| \mathrm{i}=1,2, \ldots, \mathrm{m}\right)$ e os passos primais e duais respectivamente. Para o método FLBR o tempo de processamento foi de 0,12 segundos. A Tabela 6 mostra um resumo do processo de convergência do método PDBL com tempo de processamento de 0,15 segundos. A solução inicial do sistema CESP 53 barras foi a mesma para os dois métodos. O parâmetro de barreira inicial foi $\mu=0,01$ e $\mu=0,001$ para os métodos FLBR e PDBL, respectivamente.

Tabela 5 - Convergência do método FLBR aplicado ao sistema CESP 53 barras.

\begin{tabular}{|c||c||c||c||c||}
\hline It. & F. Ob. & Erro & $\boldsymbol{\alpha}_{\mathbf{p}}$ & $\boldsymbol{\alpha}_{\mathbf{d}}$ \\
\hline \hline 0 & 2,289 & 8,465 & - & - \\
\hline \hline 1 & 2,145 & 0,201 & 0,95 & 0,95 \\
\hline \hline 2 & 2,083 & 0,049 & 1,00 & 1,00 \\
\hline \hline 3 & 2,037 & 0,069 & 1,00 & 1,00 \\
\hline \hline 4 & 2,034 & $6,0 \mathrm{e}-4$ & 1,00 & 1,00 \\
\hline
\end{tabular}

Tabela 6 - Convergência do método PDBL aplicado ao sistema CESP 53 barras.

\begin{tabular}{||c||c||c||c||c||}
\hline It. & F. Ob. & Erro & $\boldsymbol{\alpha}_{\mathbf{p}}$ & $\boldsymbol{\alpha}_{\mathbf{d}}$ \\
\hline \hline 0 & 2,289 & 8,465 & - & - \\
\hline \hline 1 & 2,179 & 0,433 & 1,000 & 0,580 \\
\hline \hline 2 & 2,123 & 0,020 & 1,000 & 1,000 \\
\hline \hline 3 & 2,063 & 0,017 & 1,000 & 1,000 \\
\hline \hline 4 & 2,032 & 0,012 & 1,000 & 1,000 \\
\hline \hline 5 & 2,037 & $1,0 \mathrm{e}-4$ & 1,000 & 1,000 \\
\hline
\end{tabular}

Destaca-se das Tabelas 5 e 6 que para o sistema CESP 53 barras o método da FLBR em relação PDBL convergiu com uma iteração a menos, com o mesmo tempo de processamento por iteração. Para os dois métodos o estado final do sistema CESP 53 barras apresenta duas magnitudes de tensão próximas aos seus limites superiores e todas as restrições canalizadas de tensão, tap e de potência reativa estão dentro de seus limites. A solução final obedece às restrições do sistema, satisfazendo o critério de parada com as seguintes tolerâncias para $\xi_{1}=10^{-3}, \xi_{2}=10^{-3}$ e $\xi_{3}=10^{-1}$ p.u, para uma potência de base de 100 MVA.

\section{C - Sistema equivalente brasileiro sul-sudeste}

O sistema equivalente brasileiro sul-sudeste possui as seguintes características: uma barra de referência; 111 barras de controle de reativo; 675 barras de carga; 1065 linhas de transmissão e 86 transformadores com taps variáveis.

O problema de DOR associado ao sistema brasileiro com 787 barras é composto por uma função objetivo, 1461 equações não-lineares, 111 inequações não-lineares canalizadas de 
potência reativa, 86 restrições canalizadas de tap e 787 restrições canalizadas de tensão. Com um total de 9025 variáveis e uma matriz Hessiana da função Lagrangiana de ordem 7056.

As Tabelas 7 e 8 apresentam um resumo do processo de convergência do sistema equivalente brasileiro sul-sudeste para os métodos FLBR e PDBL, respectivamente. A solução inicial do sistema foi a mesma para os dois métodos. O parâmetro de barreira inicial foi $\mu=0,001 \mathrm{e}$ $\mu=0,001$ e o tempo de processamento foi de 7,05 e 13,11 segundos para os métodos FLBR e PDBL, respectivamente.

Tabela 7 - Convergência do método FLBR aplicado ao sistema sul-sudeste.

\begin{tabular}{|c||c||c||c||c||}
\hline It. & F. Ob. & Erro & $\boldsymbol{\alpha}_{\mathbf{p}}$ & $\boldsymbol{\alpha}_{\mathbf{d}}$ \\
\hline \hline 0 & 18,155 & 35,998 & - & - \\
\hline \hline 1 & 17,585 & 24,414 & 0,320 & 0,210 \\
\hline \hline 2 & 17,114 & 13,119 & 0,430 & 0,450 \\
\hline \hline 3 & 16,987 & 6,263 & 0,520 & 0,240 \\
\hline \hline 5 & 16,953 & 3,769 & 0,390 & 0,550 \\
\hline \hline 6 & 17,004 & 1,060 & 0,720 & 0,510 \\
\hline \hline 7 & 16,736 & 0,313 & 0,680 & 0,510 \\
\hline \hline 8 & 16,578 & 0,102 & 0,670 & 0,610 \\
\hline \hline 9 & 16,360 & 0,010 & 0,930 & 0,800 \\
\hline \hline
\end{tabular}

Tabela 8 - Convergência do método PDBL aplicado ao sistema sul-sudeste.

\begin{tabular}{|c||c||c||c|c||}
\hline It. & F. Ob. & Erro & $\boldsymbol{\alpha}_{\mathbf{p}}$ & $\boldsymbol{\alpha}_{\mathbf{d}}$ \\
\hline \hline 0 & 18,155 & 35,998 & - & - \\
\hline \hline 1 & 17,948 & 35,111 & 0,02 & 0,06 \\
\hline \hline 2 & 17,458 & 30,468 & 0,13 & 0,00 \\
\hline \hline 3 & 17,083 & 22,728 & 0,25 & 0,15 \\
\hline \hline 4 & 16,810 & 12,654 & 0,44 & 0,01 \\
\hline \hline 5 & 16,855 & 11,460 & 0,09 & 0,02 \\
\hline \hline 6 & 16,938 & 10,656 & 0,07 & 0,00 \\
\hline \hline 7 & 16,766 & 10,617 & 0,02 & 0,00 \\
\hline \hline 8 & 16,676 & 10,426 & 0,07 & 0,00 \\
\hline \hline 9 & 16,339 & 2,881 & 0,70 & 0,03 \\
\hline \hline 10 & 16,315 & 1,576 & 0,45 & 0,08 \\
\hline \hline 11 & 16,307 & 0,508 & 0,66 & 0,00 \\
\hline \hline 12 & 16,321 & 0,337 & 0,33 & 0,00 \\
\hline \hline 13 & 16,298 & 0,177 & 0,47 & 0,01 \\
\hline \hline 14 & 16,397 & 0,066 & 0,63 & 0,00 \\
\hline \hline 15 & 16,311 & 0,002 & 1,00 & 0,00 \\
\hline \hline 16 & 16,310 & 0,001 & 1,00 & 0,00 \\
\hline
\end{tabular}


Observa-se que para o método FLBR o problema convergiu em 9 iterações, com 24 restrições de desigualdade de magnitudes de tensão e 5 restrições de desigualdade de potência reativa ativas na solução.

Analisando o processo de convergência do sistema equivalente brasileiro sul-sudeste para os dois métodos, conclui-se que o método da FLBR teve um melhor desempenho que o PDBL quanto ao número de iterações e tempo de processamento.

Para os dois métodos o estado final do sistema equivalente brasileiro sul-sudeste apresenta todas as restrições canalizadas de tensão, tap e de potência reativa dentro de seus limites, e a solução final obedece às restrições do sistema, satisfazendo o critério de parada com as seguintes tolerâncias para $\xi_{1}=10^{-2}, \xi_{2}=10^{-2} \mathrm{e} \xi_{3}=10^{-3}$ p.u, para uma potência de base de 100 MVA.

Ressalta-se que, o método da FLBR aplicado a um problema de otimização ou ao DOR convergirá desde que a solução inicial obedeça a condição de relaxação $(\mathrm{s}>-\mu)$, as estimativas dos multiplicadores de Lagrange $\rho$ iniciais sejam positivas e o parâmetro de barreira $\mu$ definido corretamente para o problema.

\section{Conclusões}

O artigo apresenta a resolução do problema de despacho ótimo de reativos pelo método da função Lagrangiana-barreira relaxada. Essa abordagem é baseada na associação dos métodos de barreira modificada e PDBL. A condição necessária de primeira-ordem da função Lagrangiana barreira relaxada é resolvida pelo método de Newton.

O método da FLBR apresentou um bom desempenho para os sistemas testados e no teste comparativo com o método PDBL. Além disso, destaca-se que suas principais vantagens são o processo de otimização pode iniciar-se fora da região factível do problema original e poder atingir, de fato, o limite das restrições de desigualdade.

\section{Agradecimentos}

Ao CNPq (Conselho Nacional de Desenvolvimento Científico e Tecnológico) e a FAPESP (Fundação de Amparo à Pesquisa do Estado de São Paulo) pelo apoio financeiro.

\section{Referências Bibliográficas}

(1) Akrotirianakis, I. \& Rustem, B. (2005). Convergent Interior-Point Algorithm for Nonlinear Programming. Journal of Optimization Theory and Applications, 125(3), 497-521.

(2) Baptista, E.C.; Belati, E.A. \& Costa, G.R.M. da (2004). Um Método Primal-Dual Aplicado na Resolução do Problema de Fluxo de Potência Ótimo. Pesq. Oper., 24(2), 215-226.

(3) Baptista, E.C.; Belati, E.A.; Sousa, V.A. \& Da Costa, G.R.M. (2006). Primal-Dual Logarithmic Barrier and Augmented Lagrangian Function to the Loss Minimization in Power Systems. Electric Power Components \& Systems, 32(7), 775-784.

(4) Byrd, R.H.; Nocedal, J. \& Waltz, R.A. (2003). Feasible Interior Methods Using Slacks for Nonlinear Optimization. Computational Optimization and Applications, 26, 35-61. 
(5) Breitfeld, M.G. \& Shanno, D.F. (1996). Computational experience with penalty-barrier methods for nonlinear programming. Annals of Operations Research, 62, 439-463.

(6) Christie, R.D.; Wollenberg, B.F. \& Wangensteen, I. (2000). Transmission Management in the Deregulated Environment. Proceedings of the IEEE, 88(2).

(7) Conn, A.R.; Gould, N. \& Toint, P.L. (1997). A Globally Convergent Lagrangian Barrier Algorithm for Optimization With General Inequality Constraints and Simple Bounds. Mathematics of Computation, 66(217), 261-288.

(8) Costa, G.R.M. (2002). Modified Newton method for reactive dispatching. Electrical Power \& Energy Systems, 24, 815-819.

(9) Forsgren, A.; Gill, P.E. \& Wright, M.H. (2002). Interior Methods for Nonlinear Optimization. SIAM REVIEW, 44(4), 525-597.

(10) Gill, P.E.; Murray, W.; Saunders, M.A. \& Wright, M.H. (1988). Recent Developments In Constrained Optimization. Journal of Computational and Applied Mathematics, 22(2-3), 257-270.

(11) Granville, S. (1994). Optimal Reactive Dispatch Through Interior Point Methods. IEEE Transactions on Power Systems, 9, 136-146.

(12) Hestenes, M.R. (1969). Survey Paper - Multiplier and Gradient Methods. J.O.T.A, 4(5), 303-320.

(13) Hunealt, M. \& Galiana, F.D. (1991). A Survey of the Optimal Power Flow Literature. IEEE Transactions on Power Systems, 6, 762-770.

(14) Melman, A. \& Polyak, R.A. (1996). The Newton Modified Barrier Method for QP Problems. Annals of Operations Research, 54, 465-519.

(15) Momoh, J.A.; EL-Hawary, M.E. \& Adapa, R. (1999a,b). A review of Selected Optimal Power Flow Literature to 1993 - Part I: Nonlinear and Quadratic Programming and Approaches - Part II: Newton, Linear Programming and Interior Point Methods. IEEE Transactions on Power Systems, 14(1), 96-111.

(16) Monteiro, R.D.C. \& Adler, I. (1989a). Interior Path Following Primal-Dual Algorithms - Part I: Linear Programming. Mathematical Programming, 44(1), 27-41.

(17) Monteiro, R.D.C. \& Adler, I. (1989b). Interior Path Following Primal-Dual Algorithms - Part II: Convex Quadratic Programming. Mathematical Programming, 44(1), 43-66.

(18) Monticelli, A. (1983). Fluxo de Carga em Redes de Energia Elétrica. Editora Edgard Blucher Ltda., São Paulo, Cap. 5, 75-100.

(19) Nocedal, J. \& Wright, S. (1999). Numerical Optimization. Springer-Verlag, New York.

(20) Polyak, R.A. (1992). Modified Barrier Functions. Mathematical Programming, 54(2), 177-222.

(21) Powell, M.J.D. (1969). A Method for Nonlinear Constraints in Minimization Problems. In: Optimization [edited by R. Fletcher], Academic Press, New York, 283-298.

(22) Ramos, J.L.M.; Expósito, A.G. \& Quintana, V.H. (2005). Transmission power loss reduction by interior-point methods: implementation issues and practical experience. IEE Proc.-Generation Transmission and Distribution, 152(1), 90-98. 
(23) Shanno, D.F. \& Vanderbei, R.J. (2000). Interior-point methods for nonconvex nonlinear programming: orderings and higher-order methods. Mathematical Programming, Ser. B, 87, 303-316.

(24) Sousa, V.A.; Baptista, E.C. \& Costa, G.R.M. (2004). Modified Barrier Method for Optimal Power Flow Problem. IEEE Power Engineering Society General Meeting. CD Rom ISBN 0-7803-8466-0. Denver - CO, USA.

\section{Apêndice}

\section{A - Função Barreira Modificada}

O desenvolvimento da FBM é apresentado, para esse propósito considere o seguinte problema de programação não-linear.

Minimizar $\mathrm{f}(\mathrm{x})$

sujeito a: $h_{j}(x) \geq 0, \quad j=1, \ldots, r$

em que $x \in \mathfrak{R}^{\mathrm{n}}$ representa o vetor das variáveis do problema e as funções $f$ e $h_{j}, j=1, \ldots, r$, são duas vezes continuamente diferenciáveis.

A fim de obter a definição da FBM o problema (23) passará por várias modificações. Primeiro, o parâmetro de barreira é adicionado à restrição de desigualdade.

$$
\begin{array}{ll}
\text { Minimizar } & \mathrm{f}(\mathrm{x}) \\
\text { sujeito a } & \mu+\mathrm{h}(\mathrm{x}) \geq \mu
\end{array}
$$

Segundo, a restrição de desigualdade é dividida e elevada pelo parâmetro de barreira.

Minimizar $\mathrm{f}(\mathrm{x})$

sujeito a $[1+(\mathrm{h}(\mathrm{x}) / \mu)]^{\mu} \geq(1)^{\mu}$

Na seqüência a função $\ln ($.) é aplicada a restrição de desigualdade, resultando em:

$$
\begin{array}{ll}
\text { Minimizar } & \mathrm{f}(\mathrm{x}) \\
\text { sujeito a } & \mu \ln [1+(\mathrm{h}(\mathrm{x}) / \mu)] \geq 0
\end{array}
$$

Uma função Lagrangiana é associada ao problema, desta forma a FBM é definida.

$$
F(x, \mu, \rho)=\left\{\begin{array}{cc}
f(x)-\rho \mu \ln [1+(h(x) / \mu)] \text { for } h(x) \geq-\mu \\
\infty & \text { caso contrário }
\end{array}\right.
$$

\section{B - Regra para Atualização da Estimativa do Multiplicador de Lagrange}

Para atualizar as estimativas dos multiplicadores de Lagrange $\rho$, adota-se o esquema proposto por Polyak (1992). Com esse objetivo, constrói-se a FBM associada ao problema (23), como segue:

$$
F=f(x)-\rho \mu \ln \left(\mu^{-1} h(x)+1\right)
$$


e a função Lagrangiana clássica associada ao problema (23):

$$
L=f(x)-\rho h(x)
$$

A seguir, determinam-se os respectivos gradientes das equações (28) e (29):

$$
\begin{aligned}
& \nabla \mathrm{F}=\nabla \mathrm{f}(\mathrm{x})-\left[\rho /\left(\mu^{-1} \mathrm{~h}(\mathrm{x})+1\right)\right] \nabla \mathrm{h}(\mathrm{x}) \\
& \nabla \mathrm{L}=\nabla \mathrm{f}(\mathrm{x})-\rho \nabla \mathrm{h}(\mathrm{x})
\end{aligned}
$$

Seja $\mathrm{x}^{\mathrm{k}+1}$ um ponto que é solução do método de Newton, sistema (14), e que é usado para atualizar as estimativas dos multiplicadores de Lagrange. Aplicando-se as condições necessárias de primeira ordem na função (28), em relação à $\mathrm{x}^{\mathrm{k}+1}, \operatorname{com} \rho^{\mathrm{k}} \mathrm{e} \mu^{\mathrm{k}}$ constantes, com uma iteração de atraso (ou seja, utiliza-se $\mathrm{x}^{\mathrm{k}+1}$ para atualizá-los), obtém-se:

$$
\nabla \mathrm{f}\left(\mathrm{x}^{\mathrm{k}+1}\right)=\left[\rho^{\mathrm{k}} \mu^{\mathrm{k}} /\left(\mathrm{h}\left(\mathrm{x}^{\mathrm{k}+1}\right)+\mu^{\mathrm{k}}\right)\right] \nabla \mathrm{h}\left(\mathrm{x}^{\mathrm{k}+1}\right)
$$

Agora, aplicando as condições necessárias de primeira ordem e substituindo $\mathrm{x}^{\mathrm{k}+1}$ em (31), mas após ter ocorrido a atualização de $\mu$ e $\rho$, tem-se:

$$
\nabla \mathrm{f}\left(\mathrm{x}^{\mathrm{k}+1}\right)=\rho^{\mathrm{k}+1} \nabla \mathrm{h}\left(\mathrm{x}^{\mathrm{k}+1}\right)
$$

Finalmente, comparando (32) e (33), chega-se no seguinte esquema para atualizar a estimativa do multiplicador de Lagrange associado a FBM:

$$
\rho^{\mathrm{k}+1}=\rho^{\mathrm{k}} \mu^{\mathrm{k}} /\left[\mathrm{h}\left(\mathrm{x}^{\mathrm{k}+1}\right)+\mu^{\mathrm{k}}\right]
$$

\section{C - Problema de Despacho Ótimo de Reativos}

O problema de DOR apresentado em (7) pode ser reescrito utilizando as equações do fluxo de potência, a função objetivo e as demais restrições, conforme Monticelli (1983).

$$
\begin{array}{lll}
\text { Minimizar } & \sum_{(\mathrm{k}, \mathrm{m}) \in \mathrm{NL}} \mathrm{f}_{\mathrm{km}}(\mathrm{t}, \mathrm{V}, \theta) & \\
\text { sujeito a } & \Delta \mathrm{P}_{\mathrm{i}}(\mathrm{t}, \mathrm{V}, \theta)=0 & \mathrm{i}=1, \ldots, \mathrm{NBCCR} \\
& \Delta \mathrm{Q}_{\mathrm{j}}(\mathrm{t}, \mathrm{V}, \theta)=0 & \mathrm{j}=1, \ldots, \mathrm{NBC} \\
& \underline{\mathrm{Q}}_{1} \leq \mathrm{Q}_{1}(\mathrm{t}, \mathrm{V}, \theta) \leq \overline{\mathrm{Q}}_{1} & \mathrm{l}=1, \ldots, \mathrm{NBCR} \\
& \underline{\mathrm{t}}_{\mathrm{o}} \leq \mathrm{t}_{\mathrm{o}} \leq \overline{\mathrm{t}}_{\mathrm{o}} & \mathrm{o}=1, \ldots, \mathrm{NT} \\
& \underline{\mathrm{V}}_{\mathrm{p}} \leq \mathrm{V}_{\mathrm{p}} \leq \overline{\mathrm{V}}_{\mathrm{p}} & \mathrm{p}=1, \ldots, \mathrm{NB}
\end{array}
$$

em que:

- V e $\theta$ são os vetores da magnitude e fase da tensão respectivamente;

- t é o tap do transformador;

- $\mathrm{f}_{\mathrm{km}}(\mathrm{t}, \mathrm{V}, \theta)$ é a função de perdas de potência ativa na transmissão na linha km, dada por:

$$
\mathrm{f}_{\mathrm{km}}(\mathrm{t}, \mathrm{V}, \theta)=\sum_{(\mathrm{k}, \mathrm{m}) \in \mathrm{NL}} \mathrm{g}_{\mathrm{km}}\left(\mathrm{V}_{\mathrm{k}}^{2}+\mathrm{V}_{\mathrm{m}}^{2}-2 \mathrm{~V}_{\mathrm{k}} \mathrm{V}_{\mathrm{m}} \cos \theta_{\mathrm{km}}\right)
$$


as equações de balanço do sistema elétrico são dadas por:

1. Potência ativa para as barras de carga e de controle de reativo:

$$
\begin{aligned}
& \Delta \mathrm{P}_{\mathrm{k}}(\mathrm{t}, \mathrm{V}, \theta)=\mathrm{P}_{\mathrm{k}}^{\mathrm{G}}-\mathrm{P}_{\mathrm{k}}^{\mathrm{C}}-\sum_{\mathrm{m} \in \Omega} \mathrm{P}_{\mathrm{km}}(\mathrm{t}, \mathrm{V}, \theta) ; \\
& \mathrm{P}_{\mathrm{km}}(\mathrm{t}, \mathrm{V}, \theta)=\left(\mathrm{t} \mathrm{V}_{\mathrm{k}}\right)^{2} \mathrm{~g}_{\mathrm{km}}-\left(\mathrm{tV}_{\mathrm{k}}\right) \mathrm{V}_{\mathrm{m}}\left(\mathrm{g}_{\mathrm{km}} \cos \theta_{\mathrm{km}}+\mathrm{b}_{\mathrm{km}} \operatorname{sen} \theta_{\mathrm{km}}\right)
\end{aligned}
$$

2. Potência reativa para as barras de carga:

$$
\begin{aligned}
& \Delta \mathrm{Q}_{\mathrm{k}}(\mathrm{t}, \mathrm{V}, \theta)=\mathrm{Q}_{\mathrm{k}}^{\mathrm{G}}-\mathrm{Q}_{\mathrm{k}}^{\mathrm{C}}-\sum_{\mathrm{m} \in \Omega} \mathrm{Q}_{\mathrm{km}}(\mathrm{t}, \mathrm{V}, \theta) ; \\
& \mathrm{Q}_{\mathrm{km}}(\mathrm{t}, \mathrm{V}, \theta)=-\left(\mathrm{tV}_{\mathrm{k}}\right)^{2}\left(\mathrm{~b}_{\mathrm{km}}+\mathrm{b}_{\mathrm{km}}^{\mathrm{sh}}\right)+\left(\mathrm{tV}_{\mathrm{k}}\right) \mathrm{V}_{\mathrm{m}}\left(\mathrm{b}_{\mathrm{km}} \cos \theta_{\mathrm{km}}-\mathrm{g}_{\mathrm{km}} \operatorname{sen} \theta_{\mathrm{km}}\right)
\end{aligned}
$$

3. Limite na geração de potência reativa para as barras de controle de reativo:

$$
\mathrm{Q}_{\mathrm{k}}(\mathrm{t}, \mathrm{V}, \theta)=\sum_{\mathrm{m} \in \Omega}-\left(\mathrm{t} \mathrm{V}_{\mathrm{k}}\right)^{2}\left(\mathrm{~b}_{\mathrm{km}}+\mathrm{b}_{\mathrm{km}}^{\mathrm{sh}}\right)+\left(\mathrm{t} \mathrm{V}_{\mathrm{k}}\right) \mathrm{V}_{\mathrm{m}}\left(\mathrm{b}_{\mathrm{km}} \cos \theta_{\mathrm{km}}-\mathrm{g}_{\mathrm{km}} \operatorname{sen} \theta_{\mathrm{km}}\right)
$$

- $\mathrm{g}_{\mathrm{km}}, \mathrm{b}_{\mathrm{km}}$ e $\mathrm{b}_{\mathrm{km}}^{\mathrm{sh}}$ são a condutância, a susceptância e a susceptância shunt da linha, respectivamente;

- $\mathrm{P}_{\mathrm{k}}^{\mathrm{G}}$ e $\mathrm{P}_{\mathrm{k}}^{\mathrm{C}}$ são as potências ativas, geradas e consumidas, respectivamente;

- $\mathrm{Q}_{\mathrm{k}}^{\mathrm{G}}$ e $\mathrm{Q}_{\mathrm{k}}^{\mathrm{C}}$ são as potências reativas, geradas e consumidas, respectivamente;

- $\underline{Q}_{k}$ e $\bar{Q}_{k}$ são os limites mínimos e máximos de geração de potência reativa;

- $\underline{\mathrm{V}}_{\mathrm{k}}$ e $\overline{\mathrm{V}}_{\mathrm{k}}$ são os limites mínimos e máximos das magnitudes das tensões;

- $\underline{t}_{i}$ e $\bar{t}_{i}$ são os limites mínimos e máximos dos taps variáveis dos transformadores;

- $\Omega$ é o conjunto de todas as barras vizinhas à barra $\mathrm{k}$, incluindo ela mesma;

- NL é o número total de linhas de transmissão;

- NB é o número de barras do sistema elétrico;

- NBC é o número de barras de carga;

- NBCR é o número de barras de controle de reativo;

- NBCCR é o número de barras de carga e de controle de reativos;

- NT é o número de transformadores com tap variável. 